\title{
Epigenomic stability assessment during cryopreservation and physiology among various strains of Chromochloris zofingiensis (Chlorophyceae) and their genetic variability revealed by AFLP and MS-AFLP
}

\author{
Niklas Reichelt ${ }^{1} \cdot$ Dorian Leger $^{1} \cdot$ Mareike Daubert $^{1} \cdot$ Paolo Ruffino $^{2} \cdot$ Thomas Pröschold $^{3} \cdot$ Tatyana Darienko $^{1}$ (D)
}

Received: 27 July 2020 / Revised and accepted: 8 April 2021 / Published online: 2 July 2021

(C) The Author(s) 2021

\begin{abstract}
Chromochloris zofingiensis (Dönz) Fucíková \& L.A.Lewis, due to its production of highly valuable carotenoids such as astaxanthin, is a model organism in biotechnology. Since the recognition of this physiological property, many biotechnological applications have only used a single strain (SAG 211-14 = CCAP 211/14 = UTEX $32=$ ATCC 30412) to produce biomass and carotenoids. However, multiple acquisitions of strains putatively belonging to the same species raised the question of the conspecificity of those strains and their properties. In this study, the conspecificity of the available strains, which are deposited axenically in SAG, was tested using SSU and ITS rDNA sequencing and AFLP (EcoRI/PstI) analyses. The comparison of SSU and ITS rDNA sequences as well as the AFLP patterns revealed that the investigated strains formed two very similar groups, (1) SAG 211-14, SAG 4.80, SAG 31.80, and SAG 34.80 and (2) SAG 221-2. All strains belonged to one species, C. zofingiensis, and represented one monophyletic lineage within the so-called DO-group of the Chlorophyceae. The robustness to cryopreservation and the subsequent epigenetic variability was detected using the methylation-sensitive AFLP (EcoRI/MspI and EcoRI/ HpaII) among the five Chromochloris strains. All strains showed a high rate of survival (54.4-98.1\%) during cryopreservation. The methylation patterns varied between precryo and postcryo in all strains detected among three time points (before, shortly after, and 8 weeks after cryopreservation), showing that the MS-AFLP technique has the potential to detect epigenetic effects occurring in response to cryopreservation and other stresses. Finally, the potential of these five strains for usage in biotechnological applications was proven by growing them in aerated cultures with and without additional carbon dioxide supply. The comparison showed that all strains produced high amounts of biomass and carotenoids under aeration with additional $\mathrm{CO}_{2}$ and were therefore suitable in biotechnology.
\end{abstract}

Keywords Chromochloris zofingiensis · AFLP · MS-AFLP · Cryopreservation · Astaxanthin · Molecular phylogeny

Tatyana Darienko

tdarien@gwdg.de

1 Albrecht-von-Haller-Institute of Plant Sciences, Experimental Phycology and Culture Collection of Algae, Georg-August University of Göttingen, D-37073 Göttingen, Germany

2 Department of Life Sciences and Systems Biology, Mycotheca Universitatis Taurinensis (MUT), University of Turin, I-10125 Turin, Italy

3 Research Department for Limnology, Leopold-Franzens-University of Innsbruck, A-5310 Mondsee, Austria

\section{Introduction}

Microalgae are a source of numerous biologically active compounds such as oils, carotenoids, vitamins, proteins, and polysaccharides. This leads to a wide variety of biotechnological applications for the development of feed and food products. The importance of algae in medical and food industries is constantly growing. Despite the extremely high diversity of algae, the number of species used for biotechnological purposes is restricted. Therefore, strains with such properties are of special value and should be maintained in culture collections under conditions where they keep their biotechnological potential. The majority of cultures in public collections are maintained in serial transfers, which can lead to mistransfers and mutations over long periods of cultivation (Day and 
Brand 2005; Lorenz et al. 2005; Müller et al. 2007). Cryopreservation was introduced as a reliable method for the conservation of algae for long-term periods without biochemical changes (Day and Brand 2005).

Since 2018, most of the new descriptions of microalgae are based on isolated strains. These should be stored in public culture collections in metabolically inactive stage and preferably cryopreserved and serve as holotypes (Art. 40.8, ICN: For the name of a new species or infraspecific taxon published on or after 1 January 2019 of which the type is a culture, the protologue must include a statement that the culture is preserved in a metabolically inactive state, Turland et al. 2018). However, one species can be represented by many strains as well as their duplicates, and they may be stored under different cultivation conditions, have other routine transfer cycles, and also may vary in nutrient media. In this process of long-term maintenance and formation of subcultures, the potential risk by human error is not negligible. Another risk may arise from inadequate documentation of the strains used in a scientific study. For instance, Müller et al. (2005) found eleven unique genotypes among 29 strains of Chlorella vulgaris with two strains even appearing to belong to entirely different species. Furthermore, the authors highlighted the lack of exact strain information in many studies. Out of 200 publications about Chlorella, only 20 studies provided such information. These findings demonstrated the strong need to test algal strains for conspecificity. Cryopreservation, the storage in liquid or vapor phase nitrogen, presents another possibility to maintain algal strains (Day and Brand 2005; Day and Harding 2008). In addition to the reduction of human interference, cryopreservation provides an environment that, unlike the manual serial transfer, ensures a perpetuation of phenotype and genotype (Kapoore et al. 2019). However, while cryopreservation is especially used for microalgae, more complex groups such as dinoflagellates showed lower success rates in the past (Rhodes et al. 2006; Stock et al. 2018).

Chromochloris zofingiensis together with Haematococcus pluvialis Flotow em. Wille belongs to the important producers of valuable natural carotenoids such as astaxanthin, a red ketocarotenoid of high commercial value. Because of its strong pigmentation function, powerful antioxidative activity, and broad beneficial effects on human health, astaxanthin is suitable for a wide range of applications in feed, food, nutraceutical, and pharmaceutical industries. While both synthetic and natural versions of astaxanthin exist, the astaxanthin market, over US $\$ 447$ million by 2014, is met mostly by synthetic production (Panis and Carreon 2016). However, synthetic astaxanthin, due to potential toxicity of the petrochemical sources it is gained from, has not been approved for human consumption but is instead restricted to, e.g., fish feed (Panis and Carreon 2016). As a result, there is growing commercial interest in natural astaxanthin production.
Chromochloris zofingiensis can grow phototrophically, mixotrophically, and heterotrophically, is easy to be cultured and scaled up both indoors and outdoors, and achieves ultrahigh cell density. Hence, C. zofingiensis has gained attention in recent years. Previous studies on astaxanthin production in $C$. zofingiensis were done using the strain SAG 211-14 (including its duplicates in other culture collections; CCAP 211/14 = UTEX $32=$ ATCC 30412). This strain was originally isolated and described by Dönz (1934) as Chlorella zofingiensis. Phylogenetic analyses revealed that this strain is not closely related to the type species of Chlorella, C. vulgaris, and represented an own lineage within the Chlorophyceae. Interestingly, SAG 211-14 is conspecific to the authentic strain SAG 221-2 (= UTEX 56) originally assigned as Chromochloris cinnabarina described by Kol and Chodat (1934). Therefore, Fučíková and Lewis (2012) transferred SAG 211-14 to the genus Chromochloris with the designation of $C$. zofingiensis as type species because of the priority rule of ICN. Several other isolates of C. zofingiensis are available in public culture collections. However, except for SAG 211-14 and its duplicates, no investigations in terms of molecular phylogeny and physiological properties have been conducted using the other strains. Other isolates of this species are studied in several publications (Kessler 1982, 1984). Kessler and Czygan (1966) compared SAG 211-14 with two new isolates called $\mathrm{Cz} 105$ (= SAG 34.80) and $\mathrm{Cz} 106$, both later erroneously called SAG 211-14b and SAG 211-14c (for differentiation, the strain SAG 211-14 was designated as SAG 211-14a in publications of Kessler 1972, 1976, 1978, 1985, Kerfin and Kessler 1978). Fott and Novakova (1969) showed that these strains belonged to one species, Chlorella zofingiensis (now Chromochloris zofingiensis).

The aim of our study was the molecular comparison of all strains assigned as $C$. zofingiensis using SSU and ITS rDNA sequences and AFLP patterns based on EcoRI/PstI restriction, the long-term stability during cryopreservation, and the physiological properties. The effects of cryopreservation, which is essential for the long-term stability of biotechnologically useful strains, were revealed using the methylation-sensitive AFLP (MS-AFLP) method with the two restriction enzyme combinations EcoRI/HpaII and $E c o$ RI/MspI. For physiological characterization, the biomass and astaxanthin production were tested for all strains under different $\mathrm{CO}_{2}$ levels to discover their potential usage in biotechnology. With these methods, we will address the following questions: (i) Are all strains conspecific? (ii) How high is the genetic variability among the strains? (iii) Does cryopreservation have an influence on epigenetic alterations in DNA methylation? and (iv) Is the biomass and astaxanthin production of all strains similar? 


\section{Materials and methods}

\section{Strains used in this study and the phylogenetic position of Chromochloris}

All strains assigned as Chromochloris zofingiensis available in public culture collections were investigated (Table S1; Supplemental Material). The genome of strain SAG 211-14 was published by Roth et al. (2017). This strain was originally sent in 2012 to the authors and sent back to SAG in 2017. We kept this strain (called here Genome) separately from the maintained strain of the collection for molecular comparison.

To test the monophyly of Chromochloris, the SSU and ITS rDNA of additional strains were sequenced (for details about origin, see Table S1). The DNA extraction, PCR, PCR purification, and sequencing were conducted with the methods described in Darienko et al. (2016). The selected strains are close relatives to Chromochloris as demonstrated by Fučíková and Lewis (2012). The SSU and ITS-2 rDNA sequences were aligned according to their secondary structures. The ITS-1 sequences were excluded from the data set because of the extreme length variations among the investigated strains. The phylogenetic analyses were inferred using the maximum likelihood, neighbor-joining, and maximum parsimony methods implemented in PAUP 4.0a167 (Swofford 2002). The best evolutionary model was calculated with the automated model selection tool in PAUP. The settings of the best model according to the Akaike information criterion is given in the legend of Fig. 1.

\section{Genetic variability among the Chromochloris strains}

Axenity test All Chromochloris strains were cultivated on basal medium with beef extract (ESFl; medium 1a in Schlösser 1994). Before DNA extraction, all strains were tested and proven axenic. Four different organic agarized media were used for these tests: Trebouxia organic medium (TOM; Ettl and Gärtner 1995), malt peptone medium (medium 16 in Schlösser 1994), basal medium with peptone (ESP; medium $1 \mathrm{~b}$ in Schlösser 1994), and ESFl medium. The plates were examined twice for contaminations in 2 and 4 weeks using a Stemi SV 11 binocular (Zeiss) and in addition checked with an Olympus BX60 (100× magnification) microscope using DICoptics. Photographical documentation was achieved with a ProgRes C14 plus (Jenoptik) camera running ProgRes CapturePro V2.9.0.1 (Jenoptik). All cultures were cultivated at $20^{\circ} \mathrm{C}$ with the light:dark cycle of $16 \mathrm{~h}$ light and $8 \mathrm{~h}$ darkness at $50 \mu \mathrm{mol}$ photons $\mathrm{m}^{-2} \mathrm{~s}^{-1}$ illumination.

DNA extraction for AFLP approaches The extraction was performed from two-week-old cultures with an Invisorb Spin Plant Mini Kit (Stratec) in duplicates. Around $100 \mathrm{mg}$ Chromochloris cultures (fresh weight) was placed into bead beating tubes and mixed with glass beads of $212-300 \mathrm{~mm}$ in diameter and shortly vortexed. The mixtures were mechanically disrupted using a PowerLyzer 24 (MoBio) for 10s at $3800 \mathrm{rpm}$. The further steps were done according to the protocols provided by the manufacturer. The quantity of DNA was assessed with a Qubit 3.0 Fluorometer (Thermo Fisher) and the quality was proven on $2 \%$ agarose gel stained with Midori Green. Samples containing long DNA strands (20-kb fragments) with a concentration of DNA between 20-25 ng $\mu \mathrm{L}^{-1}$ were used for further investigations.

AFLP protocol We used the AFLP protocol provided by Müller et al. (2005) with small changes described below. Restriction and ligation reactions were combined in one reaction (Mannschreck et al. 2002). The combination of the restriction enzymes PstI-HF (cat.-No. R3140S) and EcoRI-HF (R3101S; all from New England Biolabs, Germany) was used in this study. Approximately $100-130 \mathrm{ng}(5.5 \mu \mathrm{L})$ of high quality, non-shared DNA were incubated with $5.5 \mu \mathrm{L}$ of following mastermix. The mastermix consisted of $0.23 \mu \mathrm{L} \mathrm{H}_{2} \mathrm{O}$, $1.10 \mu \mathrm{L}$ T4 DNA ligase buffer (10×; B0202S, New England Biolabs), $1.10 \mu \mathrm{L} \mathrm{NaCl}(0.5 \mathrm{M}), 0.55 \mu \mathrm{L}$ BSA $\left(1 \mathrm{mg} \mathrm{mL}^{-1}\right)$, $0.17 \mu \mathrm{L}$ T4 DNA ligase $\left(6 \mathrm{U}_{\mu \mathrm{L}^{-1}}\right.$; M0202S; New England Biolabs), $1 \mu \mathrm{L}$ EcoRI adaptor $(5 \mu \mathrm{M}), 1 \mu \mathrm{L}$ PstI adaptor (50 $\mu \mathrm{M}), 0.25 \mu \mathrm{L} E c o \mathrm{RI}-\mathrm{HF}\left(20 \mathrm{U} \mathrm{LL}^{-1}\right)$, and $0.1 \mu \mathrm{L}$ PstI-HF (10 $\left.\mathrm{U} \mu \mathrm{L}^{-1}\right)$. The mixture was incubated on a thermocycler for $2 \mathrm{~h}$ at $37^{\circ} \mathrm{C}$ followed by $1 \mathrm{~h}$ at room temperature. The success of the digestion was checked on a $1 \%$ agarose gel and then diluted 1:5 with sterile water.

Preselective PCR amplification Two microliters of the diluted restriction-ligation mix was combined with a mastermix containing $7.25 \mu \mathrm{L} \mathrm{H}_{2} \mathrm{O}, 2.5 \mu \mathrm{L}$ PCR buffer (5×; Bioline), $0.25 \mu \mathrm{L}$ of each EcoRI+0 $(20 \mu \mathrm{M})$ and Pst $\mathrm{I}+0(20 \mu \mathrm{M})$ primers, and $0.25 \mu \mathrm{L}$ MyTaq Polymerase ( $5 \mathrm{U} \mu \mathrm{L}^{-1}$; Bioline). The mixture was briefly centrifuged at $1000 \mathrm{rpm}$ and put into a thermocycler. The PCR reactions were performed with an initial denaturation of $5 \mathrm{~min}$ at $94^{\circ} \mathrm{C}$, followed by 20 cycles of $20 \mathrm{~s}$ at $94^{\circ} \mathrm{C}$, $30 \mathrm{~s}$ at $56^{\circ} \mathrm{C}, 120 \mathrm{~s}$ at $72^{\circ} \mathrm{C}$, and a final elongation of $10 \mathrm{~min}$ at $72^{\circ} \mathrm{C}$. The samples were checked for quality on agarose gels and were diluted 10-fold for selective amplification with water.

Selective PCR amplification $1.5 \mu \mathrm{L}$ of diluted samples from the previous step were combined with a $9.5 \mu \mathrm{L}$ mastermix containing $2.25 \mu \mathrm{L} \mathrm{H} \mathrm{H}_{2} \mathrm{O}, 2.5 \mu \mathrm{L}$ PCR buffer $(5 \times), 3 \mu \mathrm{L}$ EcoRI+A/C/G (mixture of three; $10 \mu \mathrm{M}$ ), $1.5 \mu \mathrm{L}$ Pst $\mathrm{I}+$ AC $(100 \mu \mathrm{M})$, and $0.25 \mu \mathrm{L}$ MyTaq Polymerase (5 U $\left.\mu \mathrm{L}^{-1}\right)$. The different $E c o$ RI primers were labeled with 6FAM, VIC, or NED fluorochromes (Applied Biosystems). The samples were briefly centrifuged at $1000 \mathrm{rpm}$ and transferred into a thermocycler. The selective PCR 
Fig. 1 Time points of cryopreservation and workflow of the methylation-sensitive AFLP

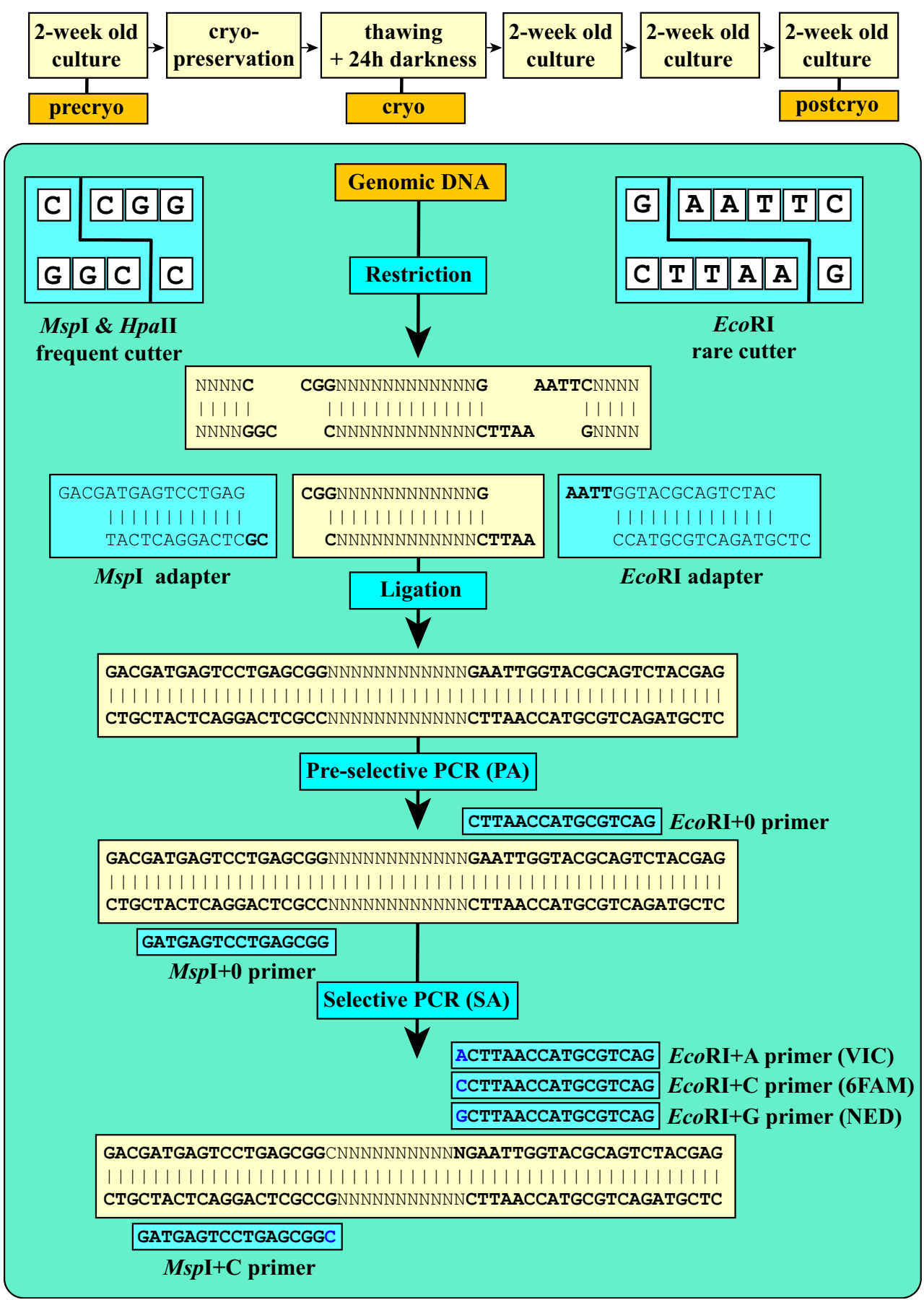

reactions were performed with an initial denaturation of $5 \mathrm{~min}$ at $95^{\circ} \mathrm{C}$, followed by 10 cycles of $20 \mathrm{~s}$ at $95^{\circ} \mathrm{C}, 30 \mathrm{~s}$ at $65^{\circ} \mathrm{C}, 120 \mathrm{~s}$ at $72^{\circ} \mathrm{C}$, followed by 20 cycles of $20 \mathrm{~s}$ at $95^{\circ} \mathrm{C}, 30 \mathrm{~s}$ at $56^{\circ} \mathrm{C}, 120 \mathrm{~s}$ at $72^{\circ} \mathrm{C}$, and a final elongation of $10 \mathrm{~min}$ at $72^{\circ} \mathrm{C}$. Afterward, the samples were diluted 10 fold. Nine microliters of Hi-Di Formamide and $0.5 \mu \mathrm{L}$ of GeneScan 600 LIZ size standard (cat. no. 4408399; Thermo Fisher) were pipetted to $0.5 \mu \mathrm{L}$ of the diluted selective amplification products.
The complete AFLP reactions were performed twice from each DNA extraction at the same time.

MS-AFLP (Fig. 1) The methylation-sensitive AFLP (MSAFLP) with the two restriction enzyme combinations EcoRI/HpaII and EcoRI/MspI was performed using the same protocol described above. For the restriction/ligation, the enzymes HpaII (10 U $\mathrm{LL}^{-1}$; R0171S) and MspI

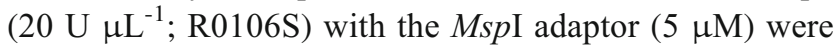


used. The primers $M s p \mathrm{I}+0(20 \mu \mathrm{M})$ and $M s p \mathrm{I}+\mathrm{C}(100$ $\mu \mathrm{M})$ were used in the preselective and selective PCR amplifications, respectively.

AFLP and MS-AFLP fragment sizing and evaluation Fluorochrome-labeled fragments were separated by capillary electrophoresis using an ABI Prism 3500 automatic sequencer (Applied Biosystems). AFLP fragments were evaluated in GeneMapper v5.0 (Thermo Fisher) and sized with GeneScan 600 LIZ size standard (Thermo Fisher). Fragments with a length of $50 \mathrm{bp}$ to $600 \mathrm{bp}$ were scored manually. The binary matrix (1 present, 0 absent) was constructed manually for AFLP (PstI). The scoring for MS-AFLP was also performed manually and the matrix contained four states ( 0 absent, $\mathbf{1}$ no methylation, both fragments $H p a \mathrm{II}$ and $M s p \mathrm{I}$ present, $\mathbf{2} \mathrm{MspI}$ present, HpaII absent, $3 \mathrm{MspI}$ absent, HpaII present; Fig. 2). The methylation scoring was done according to Schulz et al. (2013).

For the detection of fragments, the "major rule" was applied for the four replicates. For both automated and manual evaluation, thresholds of 200 fluorescent units and the form of peaks were used. The differences between two different DNA extractions and restriction-ligation products were under $2 \%$ of number of total fragments.

Data analyses The resulting matrices were exported into NEXUS format and further analyzed by PAUP 4.0a167 (Swofford 2002). The distance matrix of the binary matrix
(AFLP-PstI) was calculated in PAUP using the neighborjoining method and presented as cladogram. The support for specific nodes for the $\mathrm{NJ}$ tree was calculated with PAUP using the bootstrap method (Felsenstein 1985) with 1000 replicates (NJ and MP).

The descriptive statistics including the total number of fragments $\left(F_{\text {tot }}\right)$, the percentage of polymorphic fragments $\left(\mathrm{F}_{\text {poly }} \%\right)$, and the numbers of unique fragments $\left(\mathrm{F}_{\text {uni }}\right)$ were calculated as described in Schönswetter and Tribsch (2005).

\section{Cryopreservation and fluorescein diacetate (FDA) vital staining}

Before freezing, $750 \mu \mathrm{L}$ algal culture were mixed with $750 \mu \mathrm{L}$ of culture medium containing $10 \%$ DMSO, resulting in a final concentration of 5\% DMSO as a cryoprotectant. Samples were frozen in an ICE Cube 14S Computer Controlled Rate Freezer (SY-LAB). The protocol was conducted according to Müller et al. (2007). The tubes containing the algae with cryoprotectant with a starting temperature of $20^{\circ} \mathrm{C}$ were frozen with a rate of one degree per minute to $-40^{\circ} \mathrm{C}$. Afterward, the tubes were incubated at this temperature for a further $30 \mathrm{~min}$ and then transferred into liquid nitrogen and put into the cryotank for storage.

Cryopreserved vials were completely thawed in a water bath at $41{ }^{\circ} \mathrm{C}$ (around $3 \mathrm{~min}$ ). Afterward, vials were sterilized with $70 \%$ ethanol, and all of the samples for one strain $(0.5$ $\mathrm{mL}$ of each replicate) were transferred into $9.5 \mathrm{~mL}$ of sterile
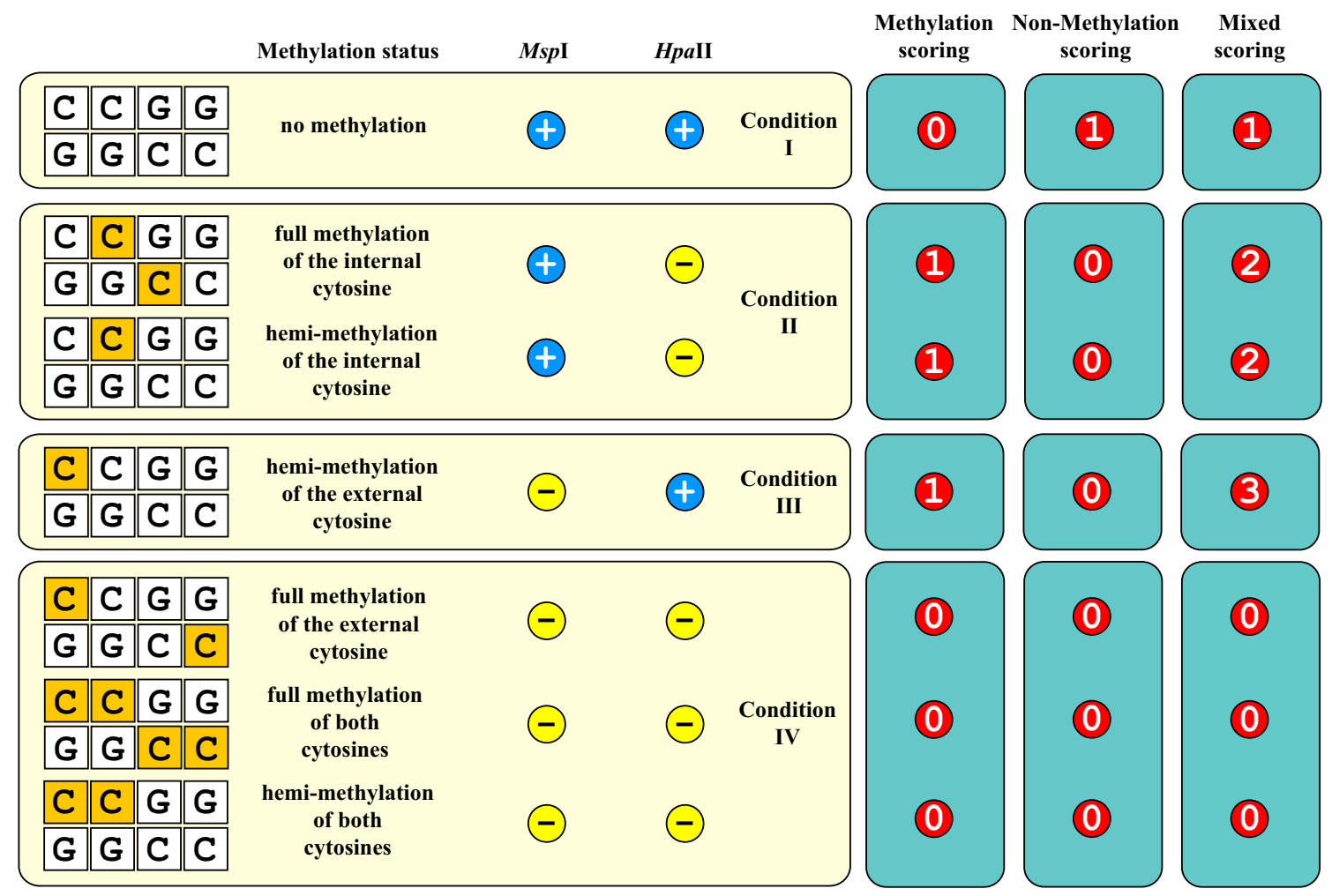

Fig. 2 Scoring types of MS-AFLP 
$\mathrm{BBM}+\mathrm{V}$ medium. The mixture was incubated in complete darkness for $24 \mathrm{~h}$. For FDA staining, a mixture was prepared by dissolving $25 \mathrm{mg}$ FDA crystals in a few drops of acetone. This mixture was filled up to $25 \mathrm{~mL}$ with methanol. One milliliter aliquots of incubated, thawed cultures were combined with $50 \mu \mathrm{L}$ FDA solution (1\% stock solution). The mixtures were incubated at room temperature for a minimum of $3 \mathrm{~min}$. Afterward, samples were examined under an Olympus BX60 fluorescence microscope (WB filter). For the calculation of the survival rates, the SAG 211-14 (Genome) strain cultured under normal conditions was used as a reference. The culture contained $80 \%$ of stained cells. This value was used for correction during the calculation of survival rates according to the following formula:

survival $[\%]=100 \times \frac{\text { total cells postcryo-dead cells postcryo }}{\text { total cells postcryo } * 0.8}$

\section{Physiological experiments}

All Chromochloris strains were pre-cultivated under standard culture conditions $\left(20^{\circ} \mathrm{C}\right.$ and a $16: 8 \mathrm{~h}$ light:dark cycle) in Erlenmeyer flasks filled with $50 \mathrm{~mL}$ of Kuhl medium (medium 12 in Schlösser 1994). After 2 weeks of cultivation, the optical density (OD) of these cultures was measured photometrically at $567 \mathrm{~nm}$ with Genesys 20 (Thermo Scientific, MA). For cultivation in aerated culturing systems, these precultures were diluted with Kuhl medium to the OD567 of approximately 0.06 to get a starting culture volume of 250 $\mathrm{mL}$. These starting cultures were cultivated in a light thermostat (Kuhl and Lorenzen 1964) under the following conditions: $20^{\circ} \mathrm{C}, 16: 8 \mathrm{~h}$ light:dark cycle, and $70-140 \mu \mathrm{mol}$ photons $\mathrm{m}^{-2} \mathrm{~s}^{-1}$. The experiments were conducted under three different conditions: aeration with pressed air without enrichment $\left(\sim 0.04 \% \mathrm{CO}_{2}\right)$, enriched with $5 \%$ or with $10 \% \mathrm{CO}_{2}$. Each treatment was done in triplicates. After the 9-day growth phase for biomass accumulation, the culture conditions were modified to induce carotenoid production in a stress phase of 7 days. The stress phase was induced by nitrogen depletion and an increase of light to $600 \mu \mathrm{mol}$ photons $\mathrm{m}^{-2} \mathrm{~s}^{-1}$. For nitrogen depletion, the biomass grown after 9 days was collected by centrifugation, and the pellets were resuspended into the $\mathrm{N}$ depleted Kuhl medium (with 1/10th nitrogen content of normal Kuhl medium). During the transfer to N-depleted medium, $20 \%$ of the biomass was separated from each sample for further analysis and storage, hence decreasing biomass prior to stress phase. These cultures were then transferred back to the light thermostat.

During the experiments, the contents of total carotenoids (Car), chlorophylls $a(\mathrm{Chl} a)$ and $b(\mathrm{Chl} b)$, as well as the biomass were measured on days $0,2,5,7,9$, and 16 .

Dry weight Dry weight (DW) was directly measured on days 9 and 16 by freeze drying and indirectly proxied with
OD correlations on days $0,2,5$, and 7 . For direct measurements, a $5 \mathrm{~mL}$ aliquot was taken from each sample, the OD at $567 \mathrm{~nm}$ was recorded, and then aliquots were placed into Eppendorf tubes. The samples were centrifuged and supernatant was discarded. The remaining pellets were frozen with liquid nitrogen briefly at $-80^{\circ} \mathrm{C}$ and then freeze dried for $24-48 \mathrm{~h}$. The DW was measured by subtracting the empty Eppendorf weight from the pellet plus Eppendorf weight and reported in $\mathrm{gDW} \mathrm{L} \mathrm{L}^{-1}$.

Pigments The pigment extraction (chlorophylls $a$ and $b$, carotenoids) method and correlation equations were based on Lichtenthaler (1987). To measure the pigments on each day, a $1 \mathrm{~mL}$ aliquot was taken from the samples and centrifuged at $14000 \times g$ for $30 \mathrm{~min}$ in a $2 \mathrm{~mL}$ tube, and the supernatant was removed. The pellets were mixed with glass beads. The samples were frozen in liquid nitrogen to enhance cell lysis. The cells were disrupted using a PowerLyzer 24 (MoBio) by $5000 \mathrm{rpm}$ for $1 \mathrm{~min}$. Lysed samples were centrifuged and cell debris partially pelleted (max rpm for 10s). Subsequently, samples were mixed with $1 \mathrm{~mL}$ acetone $(100 \%)$ and incubated on thermomixer for $20 \mathrm{~min}$ at $70^{\circ} \mathrm{C}$. After incubation, the samples were centrifuged $(14000 \mathrm{~g} \times 20 \mathrm{~min})$, and the supernatant was transferred to a spectrophotometer cuvette. The absorption at three wavelengths $(473 \mathrm{~nm}, 650 \mathrm{~nm}$, and 665 $\mathrm{nm}$ ) was measured after blanking with acetone on the same Genesys 20 used for OD. The following equations were used to quantify pigment concentrations in $\mathrm{mg} \mu \mathrm{L}^{-1}$ (see Lichtenthaler 1987):

$$
\begin{aligned}
& \text { Chl } a\left[\mathrm{mg} \mathrm{L}^{-1}\right]=11.24 \mathbf{E}_{\mathbf{6 6 5}}-2.04 \mathbf{E}_{\mathbf{6 5 0}} \\
& \text { Chl } b\left[\mathrm{mg} \mathrm{L}^{-1}\right]=20.13 \mathbf{E}_{\mathbf{6 5 0}}-4.19 \mathbf{E}_{\mathbf{6 6 5}} \\
& \text { Total Chlorophyll }\left[\mathrm{mg} \mathrm{L}^{-1}\right]=\mathrm{Chl} a+\mathrm{Chl} b \\
& \text { Total Carotenoids }\left[\mathrm{mg} \mathrm{L}^{-1}\right]=\left(1000 \mathbf{E}_{\mathbf{4 7 3}}-1.90 \mathrm{Chl} a-\right. \\
& \text { 63.14 Chl } b) / 214
\end{aligned}
$$

The cellular concentration of pigments in mg per gDW biomass was determined by dividing total $\mathrm{Chl}\left(\mathrm{mg} \mathrm{L}^{-1}\right)$ or total Car $\left(\mathrm{mg} \mathrm{L}^{-1}\right)$ by dry weight $\left(\mathrm{gDW} \mathrm{L}^{-1}\right)$. Hereafter, total chlorophyll and total carotenoids will be referred to as Chl and Car. Statistical analysis was conducted using the Tukey test with 95\% confidence intervals in Minitab 18, Minitab 18 Statistical Software (www.minitab.com).

\section{Results}

\section{Phylogenetic position and genetic variability of Chromochloris}

To test if all strains assigned as Chromochloris belong to the same monophyletic lineage, we sequenced the SSU and ITS rDNA sequences. According to Fučíková and Lewis (2012), the genera Mychonastes, Bracteacoccus, Pseudomuriella, 
Pediastrum, Planktosphaeria, and Radiosphaera are closely related to Chromochloris. Unfortunately, no ITS rDNA sequences are available for most of the genera. Therefore, we selected additional taxa of these genera and sequenced the SSU and ITS for comparison. Phylogenetic analyses of SSU and ITS-2 rDNA sequences (ITS-1 was excluded from this data set because of extreme length variations among the selected strains) revealed that most strains assigned as Chromochloris formed a monophyletic lineage within the Chlorophyceae (Fig. 3). Only two strains CAUP H6006 and CAUP H6007 were members of the genus Bracteacoccus. Interestingly, the strain CCALA 741 assigned as Chlorella homosphaera was $100 \%$ identical in SSU and ITS rDNA sequences with Chromochloris zofingiensis. All genera were highly supported in the bootstrap analyses.

All strains assigned as Chromochloris in Fig. 3 had identical SSU rDNA sequences except that the strain SAG 221-2 had an intron at position 516 according to the Escherichia coli reference. This intron was exceptionally long (1548 bp) and therefore not fully sequenced in the two GenBank entries of this strain (HQ902933 and KF673375). SAG 221-2 was the only strain which showed few variable positions in ITS rDNA sequences compared to the other strains (Fig. 4). To test the genetic variability among the Chromochloris strains, we compared the AFLP patterns using the restriction combination $E c o$ RI/PstI. The AFLP was only conducted for five strains, which were proven to be axenic in our growth test on agar plates containing three different organic culture media. In addition, the strain SAG 211-14, which was sent back to SAG by M. Roth in 2017 (herein called Genome; see Roth et al. 2017), was included to discover any changes between both strains cultivated in different laboratories. The analyses of the binary AFLP matrix (Fig. 4, Table S2; Supplemental Material) revealed that both strains were very similar (total number of
Fig. 3 Molecular phylogeny of Chromochloris and relatives based on SSU and ITS-2 rDNA sequences. The phylogenetic tree shown was inferred using the maximum likelihood method based on the data set ( 32 taxa; 2306 aligned positions) using PAUP 4.0a167. For the analysis, the best model was calculated by PAUP. The setting of the best model was given as follows: GTR+I+G (base frequencies: A 0.2350, C 0.2283 G $0.2717 \mathrm{U}$ 0.2650 ; rate matrix A-C 1.5587 , A-G 2.7084, A-U 1.5822, C-G 0.7093, C-U 4.7805, G-U 1.0000) with the proportion of invariable sites $(I=0.6337)$ and the gamma shape parameter $(\mathrm{G}=0.4250)$. The branches in bold are highly supported in all analyses (bootstrap values $>50 \%$ calculated with PAUP using maximum likelihood, neighbor-joining, and maximum parsimony)

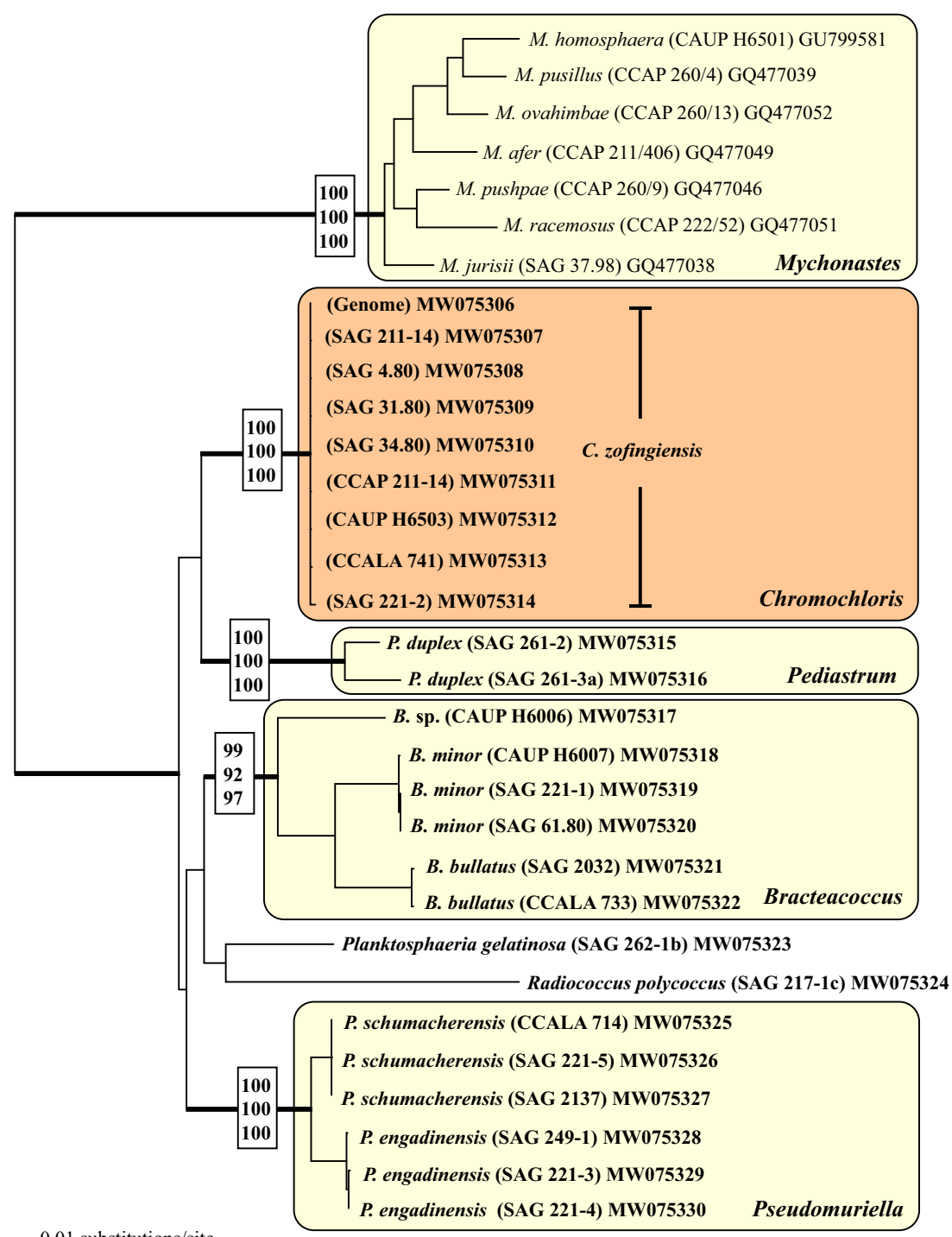

-0.01 substitutions/site 
Fig. 4 Molecular phylogeny of Chromochloris based on ITS rDNA sequences compared with the cladogram using the binary matrix revealed from AFLP approach (PstI-EcoRI)

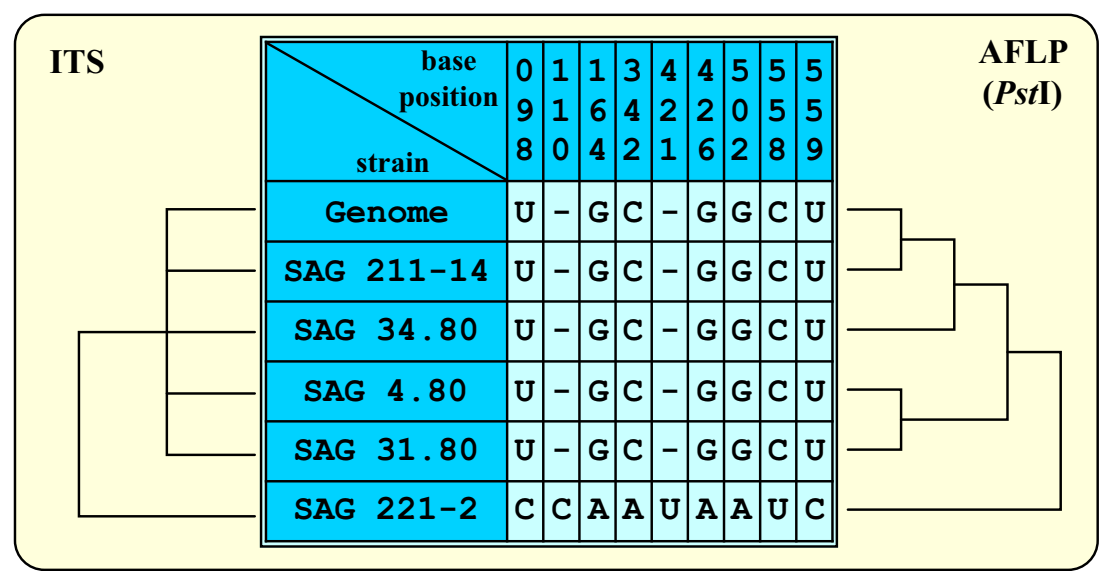

fragments $F_{\text {tot }}=240$ vs. 235 in Genome and SAG 211-14 respectively). The percentage of polymorphic fragments $\mathrm{F}_{\text {poly }} \%$ were also similar (49.08 vs. 48.06). Only the number of unique fragments $\mathrm{F}_{\text {uni }}$ varied between both strains (23 vs. 10). Other Chromochloris strains showed greater variations in the descriptive statistics (Table $\mathrm{S} 2$ ). The $\mathrm{F}_{\text {tot }}$ differed between 183 (SAG 221-2) and 250 (SAG 4.80) and $\mathrm{F}_{\text {poly }} \%$ between 37.42 and $51.12 \%$. Especially, the strain SAG 221-2 showed the highest number of unique fragments $\left(\mathrm{F}_{\text {uni }}=228\right)$.

In summary, the investigated Chromochloris strains were identical in SSU and ITS rDNA sequences and very similar in their AFLP patterns. Only the strain SAG 221-2 differed by the presence of the 516 intron in its SSU rDNA and nine base differences in its ITS rDNA as well as numerous unique fragments in its AFLP.

\section{Cryopreservation and MS-AFLP}

The use of microalgae in biotechnology requires stable and reliable strains which are always available. As highlighted above, keeping these strains maintained in serial transfers can cause problems through human errors. Therefore, we tested the robustness of the available axenic strains during cryopreservation. The strains were cryopreserved using a controlled rate freezer and 5\% DMSO as cryoprotectant. Twoweek-old cultures of each strain with a cell number of $10^{7}$ cells $\mathrm{mL}^{-1}$ were taken for cryopreservation. The survival was determined $24 \mathrm{~h}$ after thawing kept in the dark and additional $2 \mathrm{~h}$ in light. All strains except the strains SAG 4.80 and Genome had a survival rate above $80 \%$ (Table S3; Supplemental Material). However, all strains regrew well after cryopreservation and showed the typical morphology. To test the influence of cryopreservation on the methylation rate, we used MS-AFLP approach for discovery of changes in their rates. The methylation rates varied between the three time points (precryo, 2-week-old culture before cryopreservation; cryo, $24 \mathrm{~h}$ after thawing kept in darkness and $2 \mathrm{~h}$ in light; postcryo, 8 weeks after thawing with two serial transfers).
In all strains except the strain Genome, the methylation rates increased from precryo to postcryo from 20 up to $41 \%$ (Fig. 5). Only the methylation between precryo to postcryo by the strain Genome slightly decreased (31 to 28\%). The changes in methylation from precryo, cryo, to postcryo were mostly of type 2 , whereas the rate of type 3 was more less constant among the investigated strains (Fig. 5).

Not only the methylation rate differed as described, the pattern varied among the three time points. The number of polymorphic $\left(\mathrm{F}_{\text {poly }}\right)$ and unique fragments $\left(\mathrm{F}_{\text {uni }}\right)$ changed between untreated and treated samples (Table S3). The highest change in methylation was observed in strain SAG 34.80 ( $\mathrm{F}_{\text {poly }}$ 774).

Investigating the changes in the polymorphic fragments during cryopreservation in more detail, the following categories from precyro, cryo, to postcryo were chosen for comparison: (i) decrease of methylation $(0>1 / 2 / 3)$, increase of methylation $(1>0 / 2 / 3)$, and change of methylated position $(2 / 3>0 / 1)$ and (ii) rate of repairs (same methylation status of pre and postcryo), rate of changes (same methylation status of cryo and postcryo), and variable states of methylation (different states among precryo, cryo, and postcryo). The cutting sites among the polymorphic fragments had the following meanings: 0 , both cytosine are methylated; 1 , both cytosines are unmethylated; 2, only the external cytosine is methylated; and 3, only the internal cytosine is methylated. Each strain showed similar rates in changes among the polymorphic fragments (Fig. 6; Table S3). The percentage of increasing methylation varied from 38 to $49 \%$ among the strains. A higher variability could be discovered in the percentages of decreasing methylation (20-44\% among the strains). Interestingly, the changes on the external/internal methylated sites to either both methylated or both unmethylated occurred only in a rate of $15-21 \%$ with the exception of the strain Genome (33\%). The switch in methylation from type 2 to 3 or vice versa occurred only occasionally (0-11 times among the strains).

The comparison of the repairs, changes, and variable among the polymorphic fragments showed an interesting 
Fig. 5 Methylation rate of the Chromochloris strains during cryopreservation (1 no methylation, 2 internal methylation, $\mathbf{3}$ external methylation; see details in Fig. 2 and text)

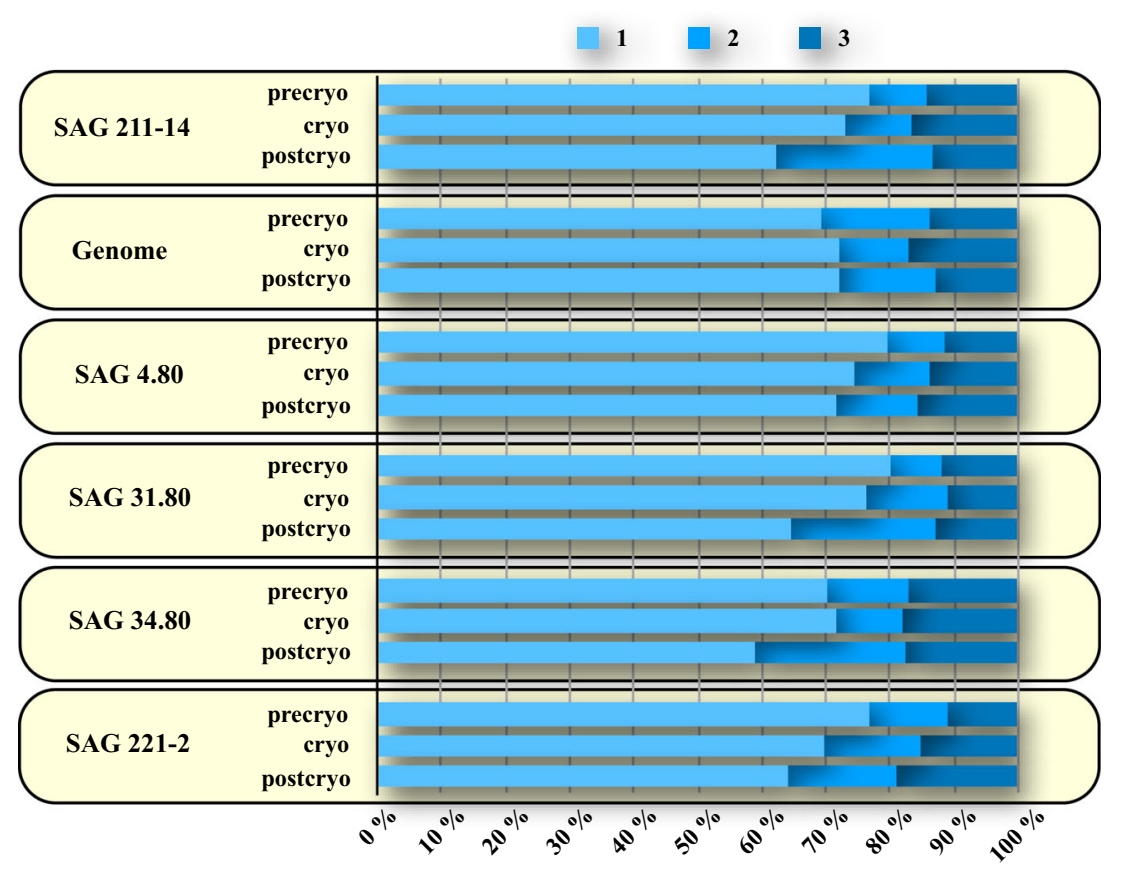

picture. The numbers of repaired fragments between precryo and postcryo were very similar among the strains (55-68) independent of the total number of polymorphic fragments. This resulted in a rate of $9-15 \%$ among the investigated strains (Fig. 6). The rates of changed (22-56\%) and variable (31$69 \%$ ) methylation states indicated that the strains did not fully recover the status of methylation after 8 weeks of regrowth.

\section{Biomass and carotenoid production under increasing carbon dioxide}

Strain SAG 211-14 has been used in many biotechnological applications concerning astaxanthin production. To investigate if the different strains of Chromochloris would also be suitable for such applications, we compared the biomass and carotenoid production under different $\mathrm{CO}_{2}$ concentrations in aerated culture systems. The strains SAG 211-14, SAG 4.80, SAG 31.80, SAG 34.80, and SAG 221-2 were grown in a two-step procedure to maximize the biomass and carotenoid content of each strain. Pre-cultures (2-week-old cultures grown in Erlenmeyer flasks) were inoculated in $250 \mathrm{~mL}$ special glass tubes (see Kuhl and Lorenzen 1964) and then cultivated for 9 days under standard conditions aerated with pressed air without additional $\mathrm{CO}_{2}$, with $5 \% \mathrm{CO}_{2}$, and with $10 \% \mathrm{CO}_{2}$ (step 1). After this time, the biomass of each culture was harvested by centrifugation and then resuspended in $250 \mathrm{~mL}$ nitrogen-depleted medium, transferred back into the culture flasks, and cultivated for a further 7 days under increased light conditions (step 2). After step 2, the cultures were harvested, and the dry weight of the biomass and the chlorophyll and carotenoid content were measured.
As demonstrated in Fig. 7, all strains showed a significant 5-10-fold higher biomass production in cultures aerated with additional $\mathrm{CO}_{2}$. The strain SAG 221-2 produced the highest biomass $\left(5.8 \mathrm{gDW} \mathrm{L}^{-1}\right)$ under $5 \%$ additional $\mathrm{CO}_{2}$. The biomass production of all other strains was between 2.4 and 4.0 gDW L ${ }^{-1}$. Despite lower biomass production being achieved at $5 \% \mathrm{CO}_{2}$ by the strains SAG 4.80, SAG 31.80, and SAG 34.80 in comparison to SAG 211-14 and SAG 221-2, the chlorophyll content was higher than that of SAG 211-14, and the amount of carotenoids was similar to that of SAG 211-14 and SAG 221-2. These results demonstrated that all strains are suitable for biotechnological applications. The biomass and pigment production increased at higher $\mathrm{CO}_{2}$ levels, but a further increase above $5 \% \mathrm{CO}_{2}$ showed no further advantages.

\section{Discussion}

\section{Molecular phylogeny and genetic variability of Chromochloris}

All strains assigned as Chromochloris formed a monophyletic lineage in the SSU/ITS-2 phylogeny (Fig. 3). Two strains (CAUP H6006 and CAUP H6007), named Chlorella zofingiensis, were members of the genus Bracteacoccus. In contrast, the strain CCALA 741 assigned as Chlorella homosphaera belonged to Chromochloris zofingiensis and was identical in sequences with the authentic strain SAG 211-14. 
Fig. 6 Changes among polymorphic fragments during cryopreservation (0 full or hemimethylation of the external or both cytosines, 1 no methylation, $\mathbf{2}$ internal methylation, $\mathbf{3}$ external methylation; see details in Fig. 2 and text)

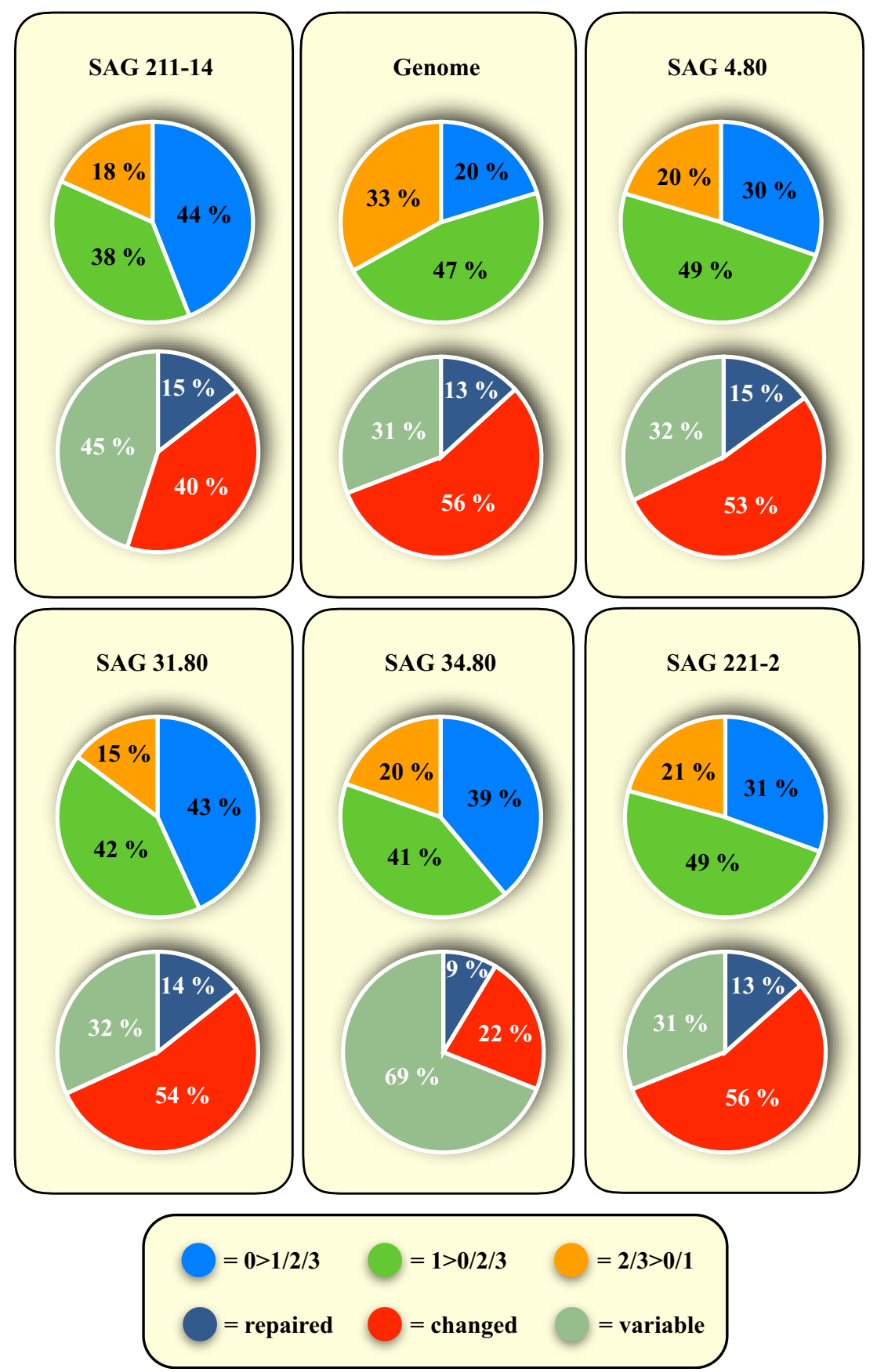

Chromochloris zofingiensis has a long history of different generic affiliations due to a lack of clear diagnostic morphological features. Since its description as Chlorella zofingiensis by Dönz (1934), this species was transferred to the genus Muriella by Hindák (1982), to Mychonastes by Kalina and Punčochářová (1987), and finally to Chromochloris by Fučíková and Lewis (2012). Phylogenetic analyses revealed that this species is almost identical in sequence with the authentic strain (SAG 221-2) of Chromochloris cinnabarina, which was described by Kol and Chodat (1934). Our analyses confirmed the findings of Fučíková and Lewis (2012).
Chromochloris is clearly separated from the genera Mychonastes, Pseudomuriella, and Bracteacoccus (Fig. 3). Fučíková and Lewis (2012) also demonstrated that one strain (ASIB S223) originally assigned as Bracteacoccus minutus belonged to Chromochloris zofingiensis. Bracteacoccus minutus was described by Schwarz (1979), which differed from Chromochloris by the production of zoospores. Unfortunately, this strain is no longer available in culture collections, and therefore the zoospore formation cannot be confirmed. We did not observe any zoospores in all studied Chromochloris strains. The relationship among these genera 

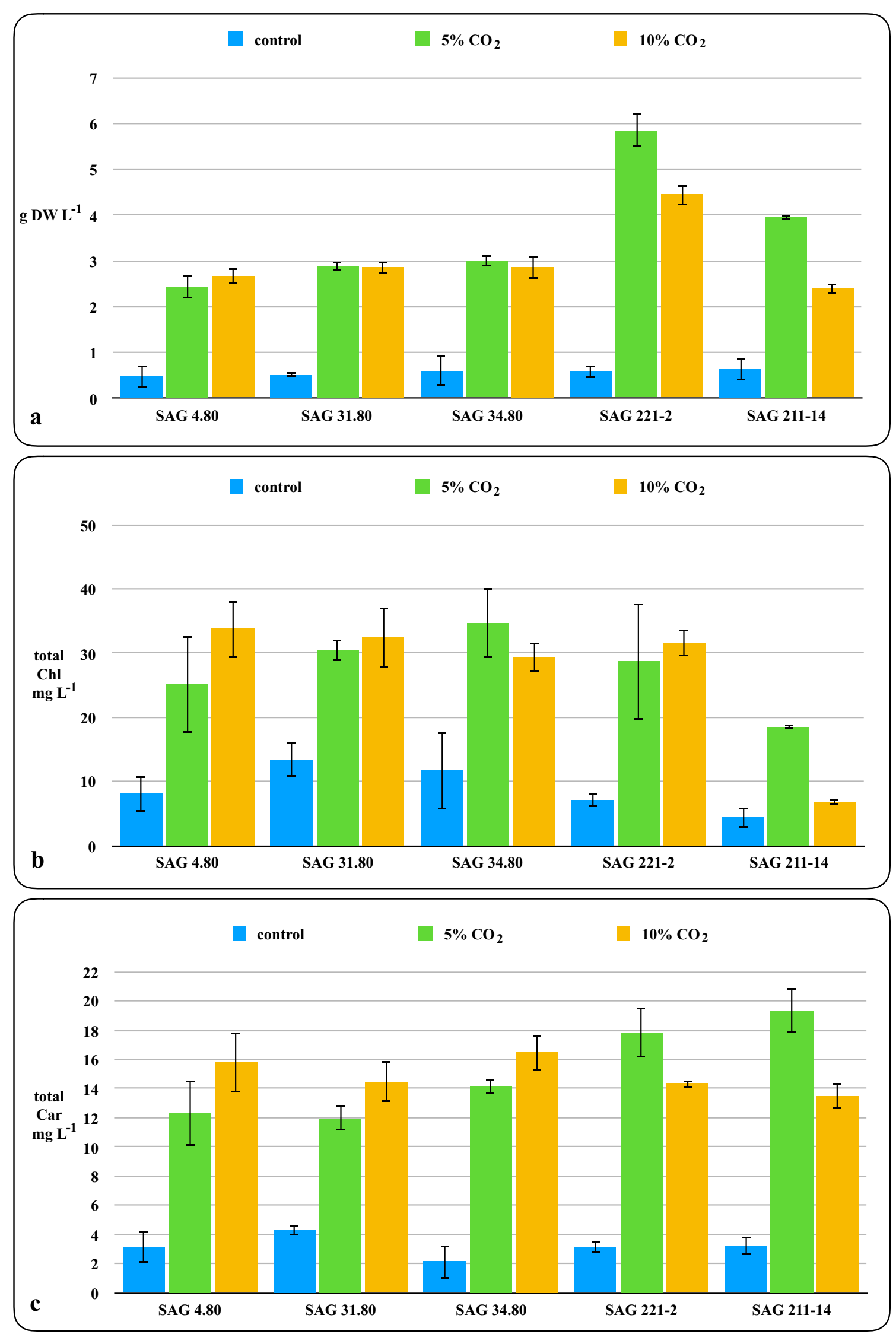

Fig. 7 Biomass (a), chlorophyll (b) and carotenoid (c) production under different aeration with $\mathrm{CO}_{2}$ 
(Chromochloris, Bracteacoccus, Pseudomuriella, Mychonastes, and others) remained unresolved and needs further studies by increased taxon sampling. Especially genome data are absent for closely related taxa. Roth et al. (2017) showed a sister relationship of Chromochloris to Monoraphidium, but this is a result that no genome sequences of other closely related taxa are available so far.

The sequence variation among $C$. zofingiensis strains was very low in SSU and ITS rDNA sequences despite the presence of the long 516 intron in SAG 221-2. This strain differed in ITS and in the AFLP pattern (EcoRI/PstI) from the other five strains (Fig. 4). Thus, the strains SAG 211-14, SAG 21114 (Genome), SAG 31.80, SAG 34.80, and SAG 4.80 seemed to be conspecific, whereas SAG 221-2 might represent a cryptic species.

\section{Epigenetic assessment of different Chromochloris strains during cryopreservation}

For the usage of microalgae in biotechnology, reliable reference strains are needed as replacement in case the bioreactors were harvested or became contaminated by other organisms. The reference microalgal strains deposited in public culture collections should be cultivated axenically without other organisms, but the maintenance via serial transfers is a potential source for contaminations by human errors. Therefore, cryopreservation may provide a potential solution for preventing such mistakes; however, the survival rate should be high, and the physiological properties should be unchanged to provide reliable material for biotechnological applications. To test the epigenetic variability during the cryopreservation, we compared the methylation patterns of several Chromochloris strains before, shortly after thawing, and after cryopreservation using the methylation-sensitive AFLP (MS-AFLP). Changes in methylation/demethylation are considered to be valuable parameters for adaptive processes in changing environments (Iqbal et al. 2011). All of the six investigated strains showed a high survival rate through the different states of cryopreservation (see Table S3). All strains showed a high number of changes in methylation. The rates increased during cryopreservation compared to precryo. Only the strain Genome showed a slight decrease in the methylation rate (Fig. 5). These results were independent of which scoring type (methylation, non-methylation, or mixed scoring; Fig. 2) was used for the analyses. However, the mixed scoring model provided more information as demonstrated in Fig. 6. For example, the comparison of the polymorphic fragments precryo and postcryo revealed around 60 fragments, which were changed shortly after cryopreservation, but switched back to the original status of precryo. This was independent from the total number of polymorphic fragments among the strains (see Table S3), indicating that the repair of changes in methylation required certain time. A third of these 60 fragments occurred at the same site, which indicated that these methylation/demethylation states of these fragments were maybe important for recovery. Interestingly, all strains, despite their good growth after cryopreservation, did not reach the same methylation states as determined in precryo samples. Therefore, it seems that microalgae need a long time (approximately 9 months estimated according to the measurements described above) to reach the same status in methylation. However, whether MS-AFLP can be generally applied as a suitable method for the detection of damages to microalgae during cryopreservation will need some further evidence from other genera and species. The MSAFLP technique has only been used for few macroalgae (the red alga Gracilariopsis lemaneiformis, Peng et al. 2018; the brown alga Saccharina japonica, Qu et al. 2013). Peng et al. (2018) studied the temperature stress response on different life stages of this red alga. They found a high level of methylation $(\sim 60 \%)$ in branches of the third generation (location of tetrasporulation). The rate increased at high temperature $\left(26^{\circ} \mathrm{C}\right)$ and decreased at low temperature $\left(15^{\circ} \mathrm{C}\right)$. Qu et al. (2013) found that chilling treatment could cause the change in DNA methylation level that may protect $S$. japonica from temperature stress by making chromosome structure tight and stable. Despite that these studies were not comparable to ours, generally the changes in methylation seem to be good indicators for adaptation to changing ecological conditions. Most studies using MS-AFLP were done for ecological epigenetics on higher plants, animals like fish, or fungi such as yeast (see review Schrey et al. 2013 and references therein).

Summarizing as demonstrated, cryopreservation has effects on the methylation pattern in all of our investigated strains, but whether these differences during cryopreservation affect the biotechnological properties among the strains, needs further investigations. Kapoore et al. (2019) compared the physiology of a marine strain (CCAP 211/21A) belonging to Chlorella vulgaris before and after cryopreservation. They found strong effects when the cultures were cryopreserved at -15 or $-80^{\circ} \mathrm{C}$ for 4 months. However, only minor effects were reported when the cultures were cryopreserved with cryoprotectant (here 5\% methanol). This indicates that the biotechnological properties do not change if the cultures were cryopreserved with optimal protocols.

\section{Biomass and carotenoid production of different Chromochloris strains in aerated cultures}

Chromochloris zofingiensis is one of the model organisms for carotenoid production in biotechnology. Many studies 
focused on the optimization of astaxanthin production under different conditions and on upscaling it in large bioreactors (Liu et al. 2014; Chen et al. 2017; Huo et al. 2018). All these applications used only one strain, SAG 211-14 or its duplicates (CCAP 211/14, UTEX 32, ATCC 30412). We compared the biomass and carotenoid production of five strains, which were available axenically in the culture collection. Our focus was not on finding the optimal conditions to maximize biomass and carotenoid content but rather on the comparison of the strains. As demonstrated above, four out of the five strains were genetically very similar (Figs. 3-4). To test if these strains also show similar properties in terms of biomass and carotenoid production, we cultivated all strains aerated with different carbon dioxide conditions in a two-step approach (see above). Our investigations demonstrated that the strain SAG 211-14 used in biotechnology did not produce the highest biomass compared to the others (Fig. 7). The strain SAG 221-2 would also be a good candidate for biotechnology. The other three strains produced a lower amount of biomass, but all strains had 5-10-fold increase in biomass under 5-10\% $\mathrm{CO}_{2}$ compared to non-enriched aeration. The optimal $\mathrm{CO}_{2}$ concentration was at $5 \%$, while an increase to $10 \%$ did not show a further increase in biomass. The amounts of chlorophyll and carotenoids were very similar among the strains, which demonstrated that all of the investigated strains are potential candidates for usage in biotechnology. Interestingly, taking into account both parameters biomass and carotenoid contents, the strains SAG 4.80, SAG 31.80, and SAG 34.80 produced a higher amount of carotenoids per dry weight in comparison to both others, which produced a higher biomass. In summary, all five strains could be used in biotechnological applications, and the choice of which strain should be used is dependent on the conditions applied and desired products. As different strains perform better under either higher or lower $\mathrm{CO}_{2}$ concentrations, this suggests the possibility of connecting the $\mathrm{CO}_{2}$-enriched air fed to algal bioreactors in a series configuration, whereby the outflow air of upstream bioreactors acts as an inflow for downstream bioreactors. During a light cycle, the $\mathrm{CO}_{2}$ concentration of air moving through the series would drop after each reactor (due to photosynthesis), such that strains with affinity for the highest $\mathrm{CO}_{2}$ treatment should be connected upstream and strains with affinity for lower $\mathrm{CO}_{2}$ concentration should be connected downstream. Such a system could reduce the loss of carbon dioxide from outlet air streams, which may be relevant for certain applications, such as in space. Further investigations are required to assess the effects of rising oxygen concentrations downstream of the series, which occurs during a light cycle dues to photosynthesis. Finally, it is worth noting that algal bioreactors connected in series would experience an inverse in gas concentrations along the series during dark cycle, i.e., rising $\mathrm{CO}_{2}$ and falling $\mathrm{O}_{2}$, due to algal respiration.
In our experiments, we reached similar biomasses among the strains compared to the published data. Del Campo et al. (2004) observed a biomass of $8 \mathrm{~g} \mathrm{~L}^{-1}$ after 25 days of growth (compared to our 3-6 g L $\mathrm{L}^{-1}$ in 10 days; Fig. 7). Our results on carotenoid production were comparable to those published in Liu et al. (2014) despite that different approaches were used in the different studies. The two-step approach applied in this study could be used for optimization of biomass and carotenoid production. Whether this approach is suitable for scaling up needs to be tested.

Supplementary Information The online version contains supplementary material available at https://doi.org/10.1007/s10811-021-02468-2.

Acknowledgements Open Access funding enabled and organized by Projekt DEAL. Our study was supported by two projects (KALT: Cryostress - adaptation mechanisms of cells toward ultra-deep temperatures funded by Leibniz Association and DSMZ-German Collection of microorganisms and cell lines and high $\mathrm{CO}_{2}$ tolerant algae: production of algal biomass from industrial $\mathrm{CO}_{2}$-rich flue gasses for biofuels and valuable compounds funded by Scientific Cooperation Niedersachsen-Israel of VolkswagenStiftung, contract no. ZN2727).

Open Access This article is licensed under a Creative Commons Attribution 4.0 International License, which permits use, sharing, adaptation, distribution and reproduction in any medium or format, as long as you give appropriate credit to the original author(s) and the source, provide a link to the Creative Commons licence, and indicate if changes were made. The images or other third party material in this article are included in the article's Creative Commons licence, unless indicated otherwise in a credit line to the material. If material is not included in the article's Creative Commons licence and your intended use is not permitted by statutory regulation or exceeds the permitted use, you will need to obtain permission directly from the copyright holder. To view a copy of this licence, visit http://creativecommons.org/licenses/by/4.0/.

\section{References}

Chen J-H, Liu L, Wei D (2017) Enhanced production of astaxanthin by Chromochloris zofingiensis in a microplate-based culture system under high light irradiation. Bioresour Technol 245A:518-529

Darienko T, Gustavs L, Pröschold T (2016) Species concept and nomenclatural changes within the genera Elliptochloris and Pseudochlorella (Trebouxiophyceae) based on an integrative approach. J Phycol 52:1125-1145

Day JG, Brand JJ (2005) Cryopreservation methods for maintaining cultures. In: Andersen RA (ed) Algal culturing techniques. Academic Press, New York, pp 165-187

Day JG, Harding K (2008) Cryopreservation of algae. In: Reed BM (ed) Plant cryopreservation: a practical guide. Springer, NY, pp 95-116

Del Campo JA, Rodriguez H, Moreno J, Vargas MA, Rivas J, Guerrero MG (2004) Accumulation of astaxanthin and lutein in Chlorella zofingiensis (Chlorophyta). Appl Microbiol Biotechnol 64:848-854 
Dönz OC (1934) Chlorella zofingiensis, eine neue Bodenalge. Ber Schweiz Bot Ges 43:127-131

Ettl H, Gärtner G (1995) Syllabus der Boden-, Luft- und Flechtenalgen. Gustav Fischer, Stuttgart

Felsenstein J (1985) Confidence limits on phylogenies: an approach using the bootstrap. Evolution 39:783-791

Fott B, Novakova M (1969) A monograph of the genus Chlorella. The freshwater species. In: Fott B (ed) Studies in phycology. Academia, Prag, pp 10-74

Fučíková K, Lewis LA (2012) Intersection of Chlorella, Muriella and Bracteacoccus: resurrecting the genus Chromochloris Kol et Chodat (Chlorophyceae, Chlorophyta). Fottea 12:83-93

Hindák F (1982) Taxonomic position of the chlorococcal alga Chlorella zofingiensis Dönz 1934 (Chlorophyceae). Algol Stud 30:13-23

Huo S, Wang Z, Zhu S, Shu Q, Zhu L, Qin L, Zhou W, Feng P, Zhu F, Yuan Z, Dong R (2018) Biomass accumulation of Chlorella zofingiensis G1 cultures grown outdoors in photobioreactors. Front Energy Res 6:49

Iqbal K, Jin S-G, Pfeifer GP, Szabo PE (2011) Reprogramming of the paternal genome upon fertilization involves genome-wide oxidation of 5-methylcytosine. Proc Natl Acad Sci 108:3642-3647

Kalina T, Punčochářová M (1987) Taxonomy of the subfamily Scotiellocystoideae Fott 1976 (Chlorellaceae, Chlorophyceae). Algol Stud 45:473-521

Kapoore RV, Huete-Ortega M, Day JG, Okurowska K, Slocombe SP, Stanley MS, Vaidyanathan S (2019) Effects of cryopreservation on viability and functional stability of an industrially relevant alga. Sci Rep 9:2093

Kerfin W, Kessler E (1978) Physiological and biochemical contributions to the taxonomy of the genus Chlorella XI. DNA hybridization. Arch Microbiol 116:97-103

Kessler E (1972) Physiologische und biochemische Beiträge zur Taxonomie der Gattung Chlorella VI. Verwertung organischer Kohlenstoff-Verbindungen. Arch Mikrobiol 85:153-158

Kessler E (1976) Comparative physiology, biochemistry, and the taxonomy of Chlorella (Chlorophyceae). Plant Syst Evol 125:129-138

Kessler E (1978) Physiological and biochemical contributions to the taxonomy of the genus Chlorella. XII. Starch hydrolysis and a key for the identification of 13 species. Arch Microbiol 119:13-16

Kessler E (1982) Chemotaxonomy in the Chlorococcales. Progr Phycol Res 1:111-135

Kessler E (1984) A general review on the contribution of chemotaxonomy to the systematics of green algae. In: Irvine DEG, John DM (eds) Systematics of the green algae, vol 27. Academic Press, London, pp 391-407

Kessler E (1985) Upper limits of temperature for growth in Chlorella (Chlorophyceae). Plant Syst Evol 151:67-71

Kessler E, Czygan F-C (1966) Chlorella zofingiensis Dönz: Isolierung neuer Stämme und ihre physiologisch-biochemischen Eigenschaften. Ber Dt Bot Ges 78:342-347

Kol E, Chodat F (1934) Quelques algues nouvelles des sols et de la neige du Parc National Suisse, Engadine. Bull Soc Bot Genève 25:250 263

Kuhl A, Lorenzen H (1964) Handling and culturing of Chlorella. In: Prescott DM (ed) Methods in cell physiology, vol 1. Academic Press, New York, pp 159-187

Lichtenthaler HK (1987) Chlorophylls and carotenoids: pigments of photosynthetic biomembranes. Methods Enzymol 148:350-382

Liu J, Sun Z, Gerken H, Liu Z, Jiang Y, Chen F (2014) Chlorella zofingiensis as an alternative microalgal producer of astaxanthin: biology and industrial potential. Mar Drugs 12:3487-3515

Lorenz M, Friedl T, Day JG (2005) Perpetual maintenance of actively metabolizing microalgal cultures. In: Andersen RA (ed) Algal culturing techniques. Academic Press, New York, pp 145-156
Mannschreck B, Fink T, Melzer A (2002) Biosystematics of selected Chara species (Charophyta) using amplified fragment length polymorphism (AFLP). Phycologia 41:657-666

Müller J, Friedl T, Hepperle D, Lorenz M, Day JG (2005) Distinction between multiple isolates of Chlorella vulgaris (Chlorophyta, Trebouxiophyceae) and testing for conspecificity using amplified fragment length polymorphism and ITS rDNA sequences. J Phycol 41:1236-1247

Müller J, Day JG, Harding K, Hepperle D, Lorenz M, Friedl T (2007) Assessing genetic stability of a range of terrestrial microalgae after cryopreservation using amplified fragment length polymorphism (AFLP). Am J Bot 94:799-808

Panis G, Carreon JR (2016) Commercial astaxanthin production derived by green alga Haematococcus pluvialis: a microalgae process model and a techno-economic assessment all through production line. Algal Res 18:175-190

Peng C, Sui Z, Zhou W, Hu Y, Mi P, Jiang M, Li X, Ruan X (2018) Analysis of DNA methylation of Gracilariopsis lemaneiformis under temperature stress using the methylation sensitive amplification polymorphism (MSAP) technique. J Ocean Univ China 17:623-631

Qu JQ, Wang X, Liu C, Li XL, Zhang XL, Liu T (2013) Preliminary analysis of DNA methylation of Saccharina japonica with MSAP. Period Ocean Univ China 43:54-59

Rhodes L, Smith J, Tervit R, Roberts R, Adamson J, Adams S, Decker M (2006) Cryopreservation of economically valuable marine microalgae in the classes Bacillariophyceae, Chlorophyceae, Cyanophyceae, Dinophyceae, Haptophyceae, Prasinophyceae, and Rhodophyceae. Cryobiology 52:152-156

Roth MS, Cokus SJ, Gallaher SD, Walter A, Lopez D, Erickson E, Endelman B, Westcott D, Larabell CA, Merchant SS, Pellegrini M, Niyogi KK (2017) Chromosome-level genome assembly and transcriptome of the green alga Chromochloris zofingiensis illuminates astaxanthin production. Proc Natl Acad Sci 114:E4296-E4305

Schlösser UG (1994) SAG-Sammlung von Algenkulturen at the University of Göttingen. Botanica Acta 107:424-429

Schönswetter P, Tribsch A (2005) Vicariaceae and dispersal in the alpine perennial Bupleurum stellatum L. (Apiaceae). Taxon 54:725-732

Schrey AW, Alvarez M, Foust CM, Kilvitis HJ, Lee JD, Liebl AL, Martin LB, Richards CL, Robertson M (2013) Ecological epigenetics: beyond MS-AFLP. Integr Comp Biol 53:340-350

Schulz B, Eckstein RL, Durka W (2013) Scoring and analysis of methylation-sensitive amplification polymorphisms for epigenetic population studies. Mol Ecol Resour 13:642-653

Schwarz K (1979) Neue Bodenalgen aus Dalmatien. Plant Syst Evol 131: 193-209

Stock W, Pinseel E, De Decker S, Sefbom J, Blommaert L, Chepurnova O, Sabbe K, Vyverman W (2018) Expanding the toolbox for cryopreservation of marine and freshwater diatoms. Sci Rep 8:4279

Swofford DL (2002) PAUP* phylogenetic analysis using parsimony (*and other methods), version 4.0b10. Sinauer Associates, Sunderland

Turland NJ, Wiersema JH, Barrie FR, Greuter W, Hawksworth DL, Herendeen PS, Knapp S, Kusber W-H, Li D-Z, Marhold K, May TW, McNeill J, Monro AM, Prado J, Price MJ, Smith GF (2018) International Code of Nomenclature for algae, fungi, and plants (Shenzhen Code) adopted by the Nineteenth International Botanical Congress Shenzhen, China, July 2017. Regnum Vegetabile 159, Koeltz Botanical Books, Glashütten

Publisher's note Springer Nature remains neutral with regard to jurisdictional claims in published maps and institutional affiliations. 